\title{
What is the true evidence for gender-related differences during plant and cut maneuvers? A systematic review
}

\author{
Anne Benjaminse $\cdot$ Alli Gokeler $\cdot$ Glenn S. Fleisig • \\ Timothy C. Sell $\cdot$ Bert Otten
}

Received: 23 February 2010/Accepted: 25 July 2010/Published online: 24 August 2010

(c) The Author(s) 2010. This article is published with open access at Springerlink.com

\begin{abstract}
Purpose Female athletes have a significantly higher risk of sustaining an anterior cruciate ligament (ACL) injury than male athletes. Biomechanical and neuromuscular factors have been reported as the main cause. The purpose of this review was to critically review results of the published literature on gender differences regarding biomechanical and neuromuscular movement patterns during plant and cutting maneuvers.

Methods MEDLINE (1966 to December 2008), EMBASE (1947 to December 2008) and CINAHL (1981 to December 2008) searches were performed. The seven studies meeting the inclusion criteria were analyzed.
\end{abstract}

A. Benjaminse $(\bowtie) \cdot$ B. Otten

Center for Human Movement Sciences, University Medical

Center Groningen, University of Groningen, Antonius

Deusinglaan 1, 9713 AV Groningen, The Netherlands

e-mail: a.benjaminse@med.umcg.nl

\section{A. Benjaminse}

SportsFieldLab Groningen, School of Sports Studies,

Hanze University Groningen, University of Applied Sciences,

Groningen, The Netherlands

A. Gokeler · B. Otten

Center for Rehabilitation, University Medical Center Groningen,

University of Groningen, Groningen, The Netherlands

\section{A. Gokeler}

Medisch Centrum Zuid, Groningen, The Netherlands

\section{G. S. Fleisig}

American Sports Medicine Institute, Birmingham, AL, USA

T. C. Sell

Neuromuscular Research Laboratory, Department of Sports Medicine and Nutrition, School of Health and Rehabilitation Sciences, University of Pittsburgh, Pittsburgh, PA, USA
Results Biomechanical gender differences were of questionable clinical relevance. Quadriceps dominance was not found in women.

Conclusion The question raises whether ACL injuries during plant and cutting maneuvers are purely gender related and whether women do have to move like men in order to reduce injury risk? Caution is warranted in making inferences as studies were heterogeneous in terms of subject and study characteristics and had low statistical power as a result of insufficient number of subjects. It is advised that future research moves beyond the isolated gender comparison and that larger sample sizes will be included. This review may aid in improving experiments to draw valid conclusions, in order to direct future ACL injury prevention programs, which might need to be more individualized.

Keywords Biomechanical control - Knee injury · Landing $\cdot$ Neuromuscular control $\cdot$ Prevention

\section{Introduction}

It has been demonstrated that in sports such as soccer, basketball and team handball, women have a 2.3-9.7 times higher risk of anterior cruciate ligament (ACL) rupture [1-3]. Approximately 38,000 ACL injuries occur in female athletics in the United States annually at an estimated medical cost of $\$ 17,00$ per injury [4]. There is a low prevalence of knee OA for individuals with isolated ACL injury (0-13\%) and a prevalence of knee OA between 21 and $48 \%$ for subjects with combined injuries [5]. The identification of risk factors and the development of prevention strategies therefore may have widespread health and economic implications. A significant amount of 
research has been conducted in order to determine strategies for injury prevention; yet, the incidence remains high $[6,7]$. It appears therefore that the success of current ACL injury prevention programs may be in part limited due to an incomplete understanding of the true mechanism of injury. Poor biomechanical and neuromuscular control of the lower limb is suggested to be the major risk factor of an ACL injury mechanism in women [8]. Others have questioned a cause-and-effect relationship between those proposed risk factors and ACL injuries [9]. Psychological, environmental and hormonal and anatomical factors still need more research [10]. The plant-and-cut movement is one of the most common non-contact ACL injury mechanisms [11-14]. Research related to this maneuver provides valuable information obtained during strenuous, sportsspecific activities. The purpose of this review is therefore to analyze the results of the literature regarding biomechanical and neuromuscular movement patterns during sidestep and cutting maneuvers, which could be used for the development of effective preventative programs to reduce ACL injuries.

\section{Materials and methods}

\section{Literature search and selection}

A literature search was performed to retrieve articles pertaining to the biomechanical and neuromuscular characteristics in healthy subjects during sidestep and crosscutting tasks. A combined search in PubMed (1966 to December 2008), EMBASE (1947 to December 2008) and CINAHL (1981 to December 2008) was performed. A combination of the following search terms was used: group (1) "sex characteristics", "sex factors", "gender bias", "sex difference"; group (2) "electromyography", "biomechanics", "neuromuscular control", group (3) "leg", "hip", "knee", "ankle" and group (4) "task performance", "side*", "step*", "cross*”, "cut*”, "task*”, "jump*" and "land*". Groups 1, 2 and 3 were MeSH (PubMed) or subheadings (CINAHL/EMBASE), while the terms in group 4 were searched for in the title or abstract. In addition, a hand search was done on the reference lists in included articles. Inclusion criteria were as follows: (1) in vivo, human analysis; (2) written in English, German or Dutch; (3) biomechanical and/or neuromuscular analysis; (4) analysis of sidestep or cut preceded by running; (5) healthy, adult subjects (mean age 18 or older); (6) gender comparative cross-sectional design and (7) kinematic, kinetic and/or electromyographic (EMG) data reported in numbers. Studies were excluded if only an abstract was available. From the title and abstract, two authors of the current review (A.B. and A.G.) independently tracked the results of the searches to identify potentially relevant manuscripts for full review. These two were in agreement on each study's inclusion or exclusion.

\section{Methodological quality assessment}

The full text of the selected studies was retrieved, and the methodological quality of the studies was independently assessed by two observers (A.B. and A.G.). Quality was assessed by scoring for these items: (1) Inclusion and exclusion criteria mentioned $(2$ points $=$ clearly defined, 1 point $=$ inadequately defined, 0 points $=$ not defined $)$. (2) Demographic information: age (mean and range, median or SD) and gender mentioned ( 2 points = clearly defined, 1 point $=$ inadequately defined, 0 points $=$ not defined). (3) Subject characteristics: activity level and sports of subject at the time of measurement reported $(2$ points $=$ clearly defined, 1 point $=$ inadequately defined, 0 points $=$ not defined). (4) Groups were comparable at baseline ( 2 points $=$ good comparability of groups or confounding adjusted for in analysis, 1 point $=$ confounding small, mentioned but not adjusted for, 0 points $=$ large potential for confounding, or not discussed). (5) Technical specification of measurements described in sufficient detail to permit replication of the test. Test device, number of trials, running speed, cutting angle $(2$ points $=$ clearly defined, 1 point $=$ inadequately defined, 0 points $=$ not defined). (6) Test-retest reliability of measurement device(s) reported ( 1 point $=$ yes, 0 points $=$ no $)$. (7) Outcome measures ( 2 points $=$ clearly defined, 1 point $=$ inadequately defined, 0 points $=$ not defined). (8) Description of power analysis (sample size justification) for detecting gender differences $(1$ point $=$ yes, 0 points $=$ no). (9) Statistical analysis $(2$ points $=$ details given, 1 point $=$ inadequately details given, 0 points $=$ no details given). (10) All included subjects measured, and if appropriate, missing data or withdrawals reported or explained and accounted for in the analysis $(2$ points $=$ described for each group separately and impact on outcomes analyzed, or missing rate less than 5\%, 1 point $=$ incomplete description/analysis, 0 points $=$ not analyzed or omission not justified). Therefore, the maximal possible score would be 18 points. The reviewers agreed on the answers to most of these questions. Disagreements were resolved by consensus of a third reviewer (G.S.F.).

Data abstraction and quantitative data synthesis

Data were extracted by the first author (A.B.). The variables of interest during the sidestep cutting maneuvers were as follows: EMG, kinematic and kinetic data of the hip, knee and ankle joints. Authors of the included studies were contacted when data were incomplete. Based on the 
number of subjects and the standard deviation (SD), an effect size (ES) calculation was conducted for each of the variables. Cohen's d values are reported as a measure of ES, where $0.2 \leq d \leq 0.5,0.5 \leq d \leq 0.8$ and $d \geq 0.8$ represent a small, moderate and large effect, respectively [15].

\section{Results}

Methodological quality and study characteristics

The searches in MEDLINE, CINAHL and EMBASE revealed, respectively, 210, 150 and 282 studies of which 85 duplicates were removed leaving 557 studies. After reading the title and abstract of these 557 studies, 16 studies were eligible for inclusion and were assessed [1631]. Based on the assessment, nine studies were excluded: three did not meet the age criteria [21-23], three failed on the criteria for data reporting [21, 24, 29], two did not meet the activity of interest $[17,19]$, two did not meet the gender criteria $[17,18]$ and one did not report our variables of interest [28]. Therefore, a total of seven studies were included in the review [16, 20, 25-27, 30, 31]. The results of the methodological quality assessment and subject and study characteristics of these seven studies are presented in Tables 1 and 2. On the methodological quality assessment scale from 0 to 18 , the mean score was 14.4 (range 12-16). Kinematic, kinetic and EMG data and the results of the ES analysis are shown in, respectively, Tables 3, 4 and 5 .

Kinematics and kinetics

\section{Hip angles}

One study showed greater peak hip flexion in women with an ES of 1.16 [26]. Both studies investigating hip abduction found smaller peak abduction of the hip for women, with the ES ranging from 0.87 to $0.90[26,30]$. One of two studies examining hip rotation found women to have significant smaller peak internal rotation with an ES of 0.82 [26].

\section{Knee angles}

No gender differences were found at initial contact (IC) for knee flexion, varus/valgus or internal/external rotation angles. In one of two studies [16, 26], men had significant greater peak knee flexion with an ES of 0.68 [26]. Two out of three studies $[16,26,30]$ found significant gender differences for peak knee valgus, however, only one of these two had a large ES, namely 0.99 [16], in which women had greater values. One out of three studies [16, 26, 30] found significant gender differences for peak rotation, in which women showed smaller peak internal rotation of the knee, ES 0.87 [26].

\section{Ankle angles}

One study examined ankle kinematics and found significantly greater peak pronation angles for women, ES -0.94 [26].

\section{Hip moments}

No significant gender differences were found for any hip kinetic variables.

\section{Knee moments}

The external peak extension moment was smaller in women, with an ES of 0.93 [31]. Two out of three studies [30-32] found greater external peak knee valgus moments for women (ES 1.06-1.30) [31, 32]. No gender differences were found for knee rotation moments. As external joint loads could potentially move a joint into a detrimental position, we have indicated the external loads in Table 4 for clarification.

\section{Neuromuscular control}

Two studies examined EMG activity [16, 20]. For the mean amplitude (\% maximum voluntary isometric contraction) measured, the vastus lateralis (VL) was more active in women for both the preparatory (ES -0.67) and the loading phase (ES -1.06). The gluteus medius was more active in the loading phase in women, with a moderate ES of -0.55 . The short-time mean frequency (STMF, measure of the mean frequency of the EMG signal over time [33]) at IC was lower in the VL (ES 0.99), the vastus medialis (VM) (ES 1.01) and biceps femoris (BF) of the women (ES 0.81 ). The STMF integrals (area under the curve) for the stance phase were lower in women for the VL (ES 1.23), the VM (ES 1.13) and the rectus femoris (ES 0.86). Lastly, the BF timing of peak total intensity occurred earlier before IC in women (ES 0.86), whereas the tibialis anterior timing of peak total intensity occurred later after IC (ES -1.21). No significant gender differences were found for the other EMG variables (STMF integrals for the prestance phase and total intensity at IC) or for the other muscles.

\section{Discussion}

Biomechanical gender differences were of questionable clinical relevance. Quadriceps dominance was not found in women during plant and cutting maneuvers. Furthermore, 
Table 1 Methodological quality assessment

\begin{tabular}{|c|c|c|c|c|c|c|c|c|}
\hline Criteria & Description scores & $\begin{array}{l}\text { McLean } \\
\text { et al. } \\
{[27]}\end{array}$ & $\begin{array}{l}\text { McLean } \\
\text { et al. } \\
{[26]}\end{array}$ & $\begin{array}{l}\text { Pollard } \\
\text { et al. } \\
{[30]}\end{array}$ & $\begin{array}{l}\text { McLean } \\
\text { et al. } \\
{[32]}\end{array}$ & $\begin{array}{l}\text { Sigward } \\
\text { et al. } \\
{[31]}\end{array}$ & $\begin{array}{l}\text { Beaulieu } \\
\text { et al. [16] }\end{array}$ & $\begin{array}{l}\text { Hanson } \\
\text { et al. } \\
{[20]}\end{array}$ \\
\hline 1 & $\begin{array}{l}\text { Inclusion and exclusion criteria specified } \\
2 \text { points = clearly defined } \\
1 \text { point }=\text { inadequately defined } \\
0 \text { points = not defined }\end{array}$ & 1 & 1 & 0 & 0 & 1 & 0 & 1 \\
\hline 2 & $\begin{array}{l}\text { Demographic information: age (mean and range, median or } \\
\text { SD) and gender mentioned } \\
2 \text { points = clearly defined } \\
1 \text { point }=\text { inadequately defined } \\
0 \text { points = not defined }\end{array}$ & 2 & 2 & 2 & 2 & 2 & 2 & 2 \\
\hline 3 & $\begin{array}{l}\text { Subject characteristics : activity level and sports of subject at } \\
\text { the time of measurement reported } \\
2 \text { points = clearly defined } \\
1 \text { point }=\text { inadequately defined } \\
0 \text { points = not defined }\end{array}$ & 2 & 0 & 2 & 2 & 2 & 2 & 2 \\
\hline 4 & $\begin{array}{l}\text { Groups were comparable at baseline } \\
2 \text { points }=\text { good comparability of groups or confounding } \\
\text { adjusted for in analysis } \\
1 \text { point }=\text { confounding small, mentioned but not adjusted for } \\
0 \text { points }=\text { large potential for confounding or not discussed }\end{array}$ & 2 & 2 & 2 & 2 & 2 & 2 & 2 \\
\hline 5 & $\begin{array}{l}\text { Technical specification of measurements described in } \\
\text { sufficient detail to permit replication of the test } \\
\text { Test device, number of trials, running speed, cutting angle } \\
2 \text { points = clearly defined } \\
1 \text { point }=\text { inadequately defined } \\
0 \text { points }=\text { not defined }\end{array}$ & 2 & 2 & 2 & 1 & 2 & 2 & 2 \\
\hline 6 & $\begin{array}{l}\text { Test retest reliability of measurement device reported } \\
1 \text { point }=\text { yes } \\
0 \text { points }=\text { no }\end{array}$ & 0 & 0 & 0 & 0 & 1 & 0 & 0 \\
\hline 7 & $\begin{array}{l}\text { Outcome measures } \\
2 \text { points = clearly defined } \\
1 \text { point }=\text { inadequately defined } \\
0 \text { points }=\text { not defined }\end{array}$ & 2 & 2 & 2 & 2 & 2 & 2 & 2 \\
\hline 8 & $\begin{array}{l}\text { Description of power analysis (sample size justification) for } \\
\text { detecting gender differences } \\
1 \text { point }=\text { yes } \\
0 \text { points }=\text { no }\end{array}$ & 0 & 1 & 1 & 0 & 0 & 0 & 0 \\
\hline 9 & $\begin{array}{l}\text { Statistical analysis } \\
2 \text { points }=\text { details given } \\
1 \text { point }=\text { inadequately details given } \\
0 \text { points }=\text { no details given }\end{array}$ & 2 & 2 & 2 & 1 & 2 & 2 & 2 \\
\hline 10 & $\begin{array}{l}\text { All included subjects measured, and if appropriate, missing } \\
\text { data or withdrawals reported or explained and accounted } \\
\text { for in the analysis } \\
2 \text { points = described for each group separately and impact } \\
\text { on outcomes analyzed or missing rate less than } 5 \% \\
1 \text { point }=\text { incomplete description/analysis } \\
0 \text { points = not analyzed or omission not justified }\end{array}$ & 2 & 2 & 2 & 2 & 2 & 2 & 2 \\
\hline \multicolumn{2}{|c|}{ Total score (maximum $=18$ points $)$} & 15 & 14 & 15 & 12 & 16 & 14 & 15 \\
\hline
\end{tabular}




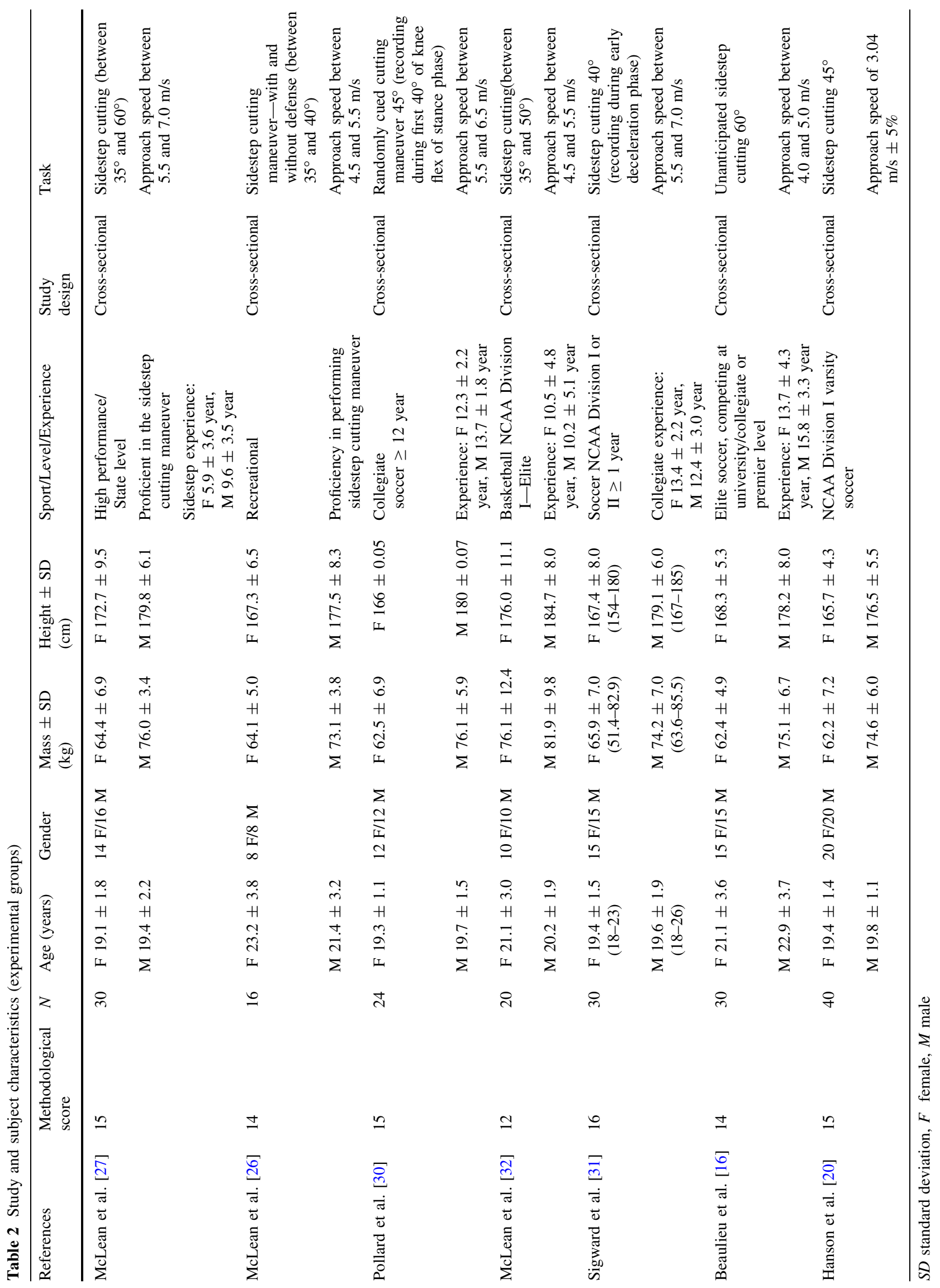


Table 3 Kinematics

\begin{tabular}{|c|c|c|c|c|c|c|}
\hline Dependent variable & Task & $\begin{array}{l}\text { Men } \\
\text { Mean } \pm \mathrm{SD}(n)\end{array}$ & $\begin{array}{l}\text { Women } \\
\text { Mean } \pm \mathrm{SD}(n)\end{array}$ & $\begin{array}{l}\text { Gender } \\
\text { difference }\end{array}$ & $P$ value & $\begin{array}{l}\text { Effect size } \\
(95 \% \mathrm{CI})\end{array}$ \\
\hline \multicolumn{7}{|l|}{ Hip angle $\left(^{\circ}\right)$} \\
\hline Peak flexion $^{a}$ & $\begin{array}{l}\text { Sidestep cutting } \\
\quad\left(\text { between } 35^{\circ} \text { and } 40^{\circ} \text { ) }\right.\end{array}$ & $54.1 \pm 11.0(8)$ & $43.2 \pm 7.5(8)$ & 10.9 & $<0.003^{*}$ & $1.16^{*}(0.04$ to 2.15$)$ \\
\hline Peak abduction $^{\mathrm{a}}$ & $\begin{array}{l}\text { Sidestep cutting } \\
\quad\left(\text { between } 35^{\circ} \text { and } 40^{\circ}\right)\end{array}$ & $33.1 \pm 8.9(8)$ & $26.7 \pm 5.5(8)$ & 6.4 & $<0.003^{*}$ & $0.87^{\star}(-0.20$ to 1.84$)$ \\
\hline Peak abduction $^{\mathrm{b}}$ & $\begin{array}{l}\text { Randomly cued cutting } \\
\left(45^{\circ}\right)\end{array}$ & $9.07 \pm 7.2(12)$ & $3.43 \pm 5.2(12)$ & 5.64 & $0.03 *$ & $0.90^{*}(-1.70$ to -0.03$)$ \\
\hline Peak internal rotation ${ }^{a}$ & $\begin{array}{l}\text { Sidestep cutting } \\
\quad\left(\text { between } 35^{\circ} \text { and } 40^{\circ} \text { ) }\right.\end{array}$ & $14.6 \pm 7.8(8)$ & $8.4 \pm 7.4(8)$ & 6.2 & $<0.003^{*}$ & $0.82^{*}(-0.24$ to 1.79$)$ \\
\hline Peak internal rotation ${ }^{\mathrm{b}}$ & $\begin{array}{l}\text { Randomly cued cutting } \\
\left(45^{\circ}\right)\end{array}$ & $3.58 \pm 8.9(12)$ & $3.37 \pm 8.5(12)$ & 0.21 & 0.98 & $0.02(-0.78$ to 0.82$)$ \\
\hline \multicolumn{7}{|l|}{ Knee angle $\left(^{\circ}\right)$} \\
\hline Flexion at $\mathrm{IC}^{\mathrm{c}}$ & $\begin{array}{l}\text { Unanticipated cutting } \\
\left(45^{\circ}\right)\end{array}$ & $15.60 \pm 6.11(15)$ & $17.95 \pm 6.76(15)$ & 2.35 & 0.326 & $-0.36(-1.08$ to 0.37$)$ \\
\hline Peak flexion $^{a}$ & $\begin{array}{l}\text { Sidestep cutting } \\
\quad\left(\text { between } 35^{\circ} \text { and } 40^{\circ} \text { ) }\right.\end{array}$ & $63.1 \pm 9.5(8)$ & $57.2 \pm 7.7(8)$ & 5.9 & $<0.003^{*}$ & $0.68(-0.36$ to 1.65$)$ \\
\hline Peak flexion ${ }^{\mathrm{c}}$ & $\begin{array}{l}\text { Unanticipated cutting } \\
\left(45^{\circ}\right)\end{array}$ & $57.36 \pm 5.01(15)$ & $57.94 \pm 7.28(15)$ & 0.58 & 0.799 & $-0.09(-0.81$ to 0.63$)$ \\
\hline $\operatorname{Varus}(+) / \operatorname{Valgus}(-)$ at $\mathrm{IC}^{\mathrm{c}}$ & $\begin{array}{l}\text { Unanticipated cutting } \\
\left(45^{\circ}\right)\end{array}$ & $1.28 \pm 6.22(15)$ & $-2.98 \pm 5.10(15)$ & 4.26 & 0.050 & $0.75(-0.01$ to 1.47$)$ \\
\hline Peak valgus $^{\mathrm{a}}$ & $\begin{array}{l}\text { Sidestep cutting } \\
\quad\left(\text { between } 35^{\circ} \text { and } 40^{\circ} \text { ) }\right.\end{array}$ & $12.1 \pm 4.5(8)$ & $14.2 \pm 5.2(8)$ & -2.1 & $<0.003^{*}$ & $-0.43(-1.40$ to 0.58$)$ \\
\hline Peak valgus ${ }^{\mathrm{b}}$ & $\begin{array}{l}\text { Randomly cued cutting } \\
\left(45^{\circ}\right)\end{array}$ & $1.53 \pm 6.0(12)$ & $2.39 \pm 3.5(12)$ & -0.86 & 0.68 & $-0.18(-0.97$ to 0.63$)$ \\
\hline Peak valgus ${ }^{c}$ & $\begin{array}{l}\text { Unanticipated cutting } \\
\left(45^{\circ}\right)\end{array}$ & $5.26 \pm 11.28(15)$ & $15.31 \pm 8.84(15)$ & 10.05 & $0.011^{*}$ & $0.99^{*}(0.21$ to 1.72$)$ \\
\hline $\begin{array}{l}\text { Internal }(+) / \text { external }(-) \\
\text { rotation at } \mathrm{IC}^{\mathrm{c}}\end{array}$ & $\begin{array}{l}\text { Unanticipated cutting } \\
\left(45^{\circ}\right)\end{array}$ & $0.17 \pm 9.27$ & $-2.70 \pm 7.26(15)$ & 2.87 & 0.354 & $0.34(-0.39$ to 1.06$)$ \\
\hline Peak internal rotation ${ }^{a}$ & $\begin{array}{l}\text { Sidestep cutting } \\
\quad\left(\text { between } 35^{\circ} \text { and } 40^{\circ} \text { ) }\right.\end{array}$ & $19.2 \pm 5.9(8)$ & $14.3 \pm 5.4(8)$ & 4.9 & $<0.003^{*}$ & $0.87^{\star}(-0.20$ to 1.84$)$ \\
\hline Peak internal rotation ${ }^{\mathrm{b}}$ & $\begin{array}{l}\text { Randomly cued cutting } \\
\left(45^{\circ}\right)\end{array}$ & $6.07 \pm 5.9(12)$ & $6.30 \pm 5.9(12)$ & -0.23 & 0.93 & $-0.04(-0.84$ to 0.76$)$ \\
\hline Peak internal rotation ${ }^{c}$ & $\begin{array}{l}\text { Unanticipated cutting } \\
\left(45^{\circ}\right)\end{array}$ & $22.91 \pm 6.92(15)$ & $19.81 \pm 5.99(15)$ & 3.1 & 0.200 & $0.48(-0.26$ to 1.19$)$ \\
\hline \multicolumn{7}{|l|}{ Ankle angle $\left(^{\circ}\right)$} \\
\hline Peak pronation $^{\mathrm{a}}$ & $\begin{array}{l}\text { Sidestep cutting } \\
\quad\left(\text { between } 35^{\circ} \text { and } 40^{\circ} \text { ) }\right.\end{array}$ & $1.5 \pm 4.9(8)$ & $7.1 \pm 6.8(8)$ & -5.6 & $<0.003 *$ & $-0.94^{*}(-1.92$ to 0.13$)$ \\
\hline
\end{tabular}

$I C$ initial contact, $S D$ standard deviation

* Significant difference $(P<0.05)$

* Large effect size (i.e. $\geq 0.80$ or $\geq-0.80$ )

${ }^{a}$ McLean et al. [26]

${ }^{b}$ Pollard et al. [30]

${ }^{c}$ Beaulieu et al. [16]

not all variables showing significant gender difference had an $\mathrm{ES} \geq 0.80$, indicating insufficient power. No differences in gender were found for knee kinematics. Overall, there is inconclusive evidence whether there are biomechanical and neuromuscular gender differences during these maneuvers.
Methodological quality

The methodological quality score ranged from 12 to 16 . A specific checklist for the current topic of interest is not available to the best of the authors' knowledge. Therefore, a combined checklist composed based on the applicable 
Table 4 Kinetics

\begin{tabular}{|c|c|c|c|c|c|c|}
\hline Dependent variable & Task & $\begin{array}{l}\text { Men } \\
\text { Mean } \pm \operatorname{SD}(n)\end{array}$ & $\begin{array}{l}\text { Women } \\
\text { Mean } \pm \mathrm{SD}(n)\end{array}$ & $\begin{array}{l}\text { Gender } \\
\text { difference }\end{array}$ & $P$ value & $\begin{array}{l}\text { Effect size } \\
(95 \% \mathrm{CI})\end{array}$ \\
\hline \multicolumn{7}{|l|}{ Hip moment $(\mathrm{Nm} / \mathrm{kg})$} \\
\hline $\begin{array}{l}\text { Internal peak abduction }^{\mathrm{a}} \\
\text { (external peak adduction) }\end{array}$ & $\begin{array}{l}\text { Randomly cued } \\
\text { cutting }\left(45^{\circ}\right)\end{array}$ & $-0.96 \pm 0.3(12)$ & $-0.98 \pm 0.4(12)$ & 0.02 & 0.74 & $0.06(-0.75$ to 0.85$)$ \\
\hline $\begin{array}{l}\text { Internal peak external rotation }{ }^{\mathrm{a}} \\
\text { (external peak internal rotation) }\end{array}$ & $\begin{array}{l}\text { Randomly cued } \\
\text { cutting }\left(45^{\circ}\right)\end{array}$ & $-0.47 \pm 0.4(12)$ & $-0.50 \pm 0.2(12)$ & 0.03 & 0.77 & $0.09(-0.71$ to 0.89$)$ \\
\hline \multicolumn{7}{|l|}{ Knee moment $(\mathrm{Nm} / \mathrm{kg})$} \\
\hline $\begin{array}{l}\text { Internal peak flexion }{ }^{\mathrm{b}} \\
\text { (external peak extension) }\end{array}$ & $\begin{array}{l}\text { Sidestep cutting } \\
\left(40^{\circ}\right)\end{array}$ & $2.1 \pm 0.8(15)$ & $1.4 \pm 0.7(15)$ & 0.7 & $0.025^{*}$ & $0.93^{*}(0.15$ to 1.66$)$ \\
\hline $\begin{array}{l}\text { Internal peak varus }{ }^{\mathrm{a}} \\
\text { (external peak valgus) }\end{array}$ & $\begin{array}{l}\text { Randomly cued } \\
\text { cutting }\left(45^{\circ}\right)\end{array}$ & $0.31 \pm 0.1(12)$ & $0.37 \pm 0.2(12)$ & -0.06 & 0.36 & $-0.38(-1.17$ to 0.44$)$ \\
\hline $\begin{array}{l}\text { Internal peak varus }{ }^{\mathrm{b}} \\
\text { (external peak valgus) }\end{array}$ & $\begin{array}{l}\text { Sidestep cutting } \\
\left(40^{\circ}\right)\end{array}$ & $0.006 \pm 0.3(15)$ & $-0.43 \pm 0.5(15)$ & 0.424 & $0.005^{*}$ & $1.06^{\star}(0.27$ to 1.79$)$ \\
\hline External peak valgus ${ }^{\mathrm{c}}$ & Sidestep cutting & $0.42 \pm 0.11(10)$ & $0.63 \pm 0.20(10)$ & -0.21 & $0.05^{*}$ & $-1.30^{*}(-2.20$ to -0.29$)$ \\
\hline $\begin{array}{l}\text { Internal peak external rotation } \\
\text { (external peak internal rotation) }\end{array}$ & $\begin{array}{l}\text { Randomly cued } \\
\text { cutting }\left(45^{\circ}\right)\end{array}$ & $-0.09 \pm 0.1(12)$ & $-0.13 \pm 0.1(12)$ & 0.04 & 0.19 & $0.40(-0.42$ to 1.19$)$ \\
\hline \multicolumn{7}{|l|}{$S D$ standard deviation } \\
\hline \multicolumn{7}{|l|}{$*$ Significant difference $(P<0.05)$} \\
\hline \multicolumn{7}{|c|}{ * $\quad$ Large effect size (i.e. $\geq 0.80$ or $\geq-0.80$ ) } \\
\hline \multicolumn{7}{|l|}{${ }^{a}$ Pollard et al. [30] } \\
\hline \multicolumn{7}{|l|}{ b Sigward et al. [31] } \\
\hline${ }^{c}$ McLean et al. [32] & & & & & & \\
\hline
\end{tabular}

items from other available checklists was made (Consort checklist, PEDro scale, QUADAS) [34-36]. It is recognized that the specific checklist used for the current review is not tested for its reliability and validity; however, the used checklists are well reported and accepted tools for quality assessments. To add more insight into the strength of the relationship between the variables of interest, the ES were also calculated. The ES ranged between -1.30 and 1.23. The studies were heterogeneous in terms of subject and study characteristics and had low statistical power as a result of insufficient number of subjects.

\section{Kinematics and kinetics}

Three studies reported on kinematics [16, 26, 30] and three on kinetics [30-32]. Nine studies (of which seven had an $\mathrm{ES} \geq 0.80$ ) reported gender differences in hip, knee and ankle kinematics, whereas eight did not find any differences. The cutting angles varied between $35^{\circ}$ and $60^{\circ}$, and the approach speed ranged from $3.04 \mathrm{~m} / \mathrm{s} \pm 5 \%$ to $5.5-7.0 \mathrm{~m} / \mathrm{s}$, which could explain in part the variability in results; an increase in approach speed of $0.3 \mathrm{~m} / \mathrm{s}$ results in a $20 \%$ increase in maximum knee valgus moment [37]. The early deceleration phase of the cutting cycle is considered the time in which the majority of non-contact ACL injuries occur [11]. This is the point in the cutting cycle where a lot of force needs to be absorbed in a short time. Sharper cutting angles require greater deceleration. Assuming that the harder the cut, the greater the peak posterior GRFs, which results in more strain on the ACL [38]. In addition, sharper cutting angles probably result in a greater chance for injury, as the amount of external tibial rotation will be greater. Increasing the amount of external tibial rotation (from $5^{\circ}$ to $13^{\circ}$ in combination with a $8^{\circ}$ valgus) results in ACL impingement [39]. This impingement mechanism was suggested as a cause in team handball players who commonly injured their ACL during valgus and external tibial rotation movements near full knee extension to moderate flexion [14]. Furthermore, lower extremity motions have been evaluated in women performing unanticipated cutting tasks with angles between $45^{\circ}$ and $90^{\circ}$ [40]. These researchers reported that hip internal rotation and knee internal rotation were increased during the $90^{\circ}$ cut compared to the $45^{\circ}$ unanticipated cut angle. Mean hip flexion was also greater in the $90^{\circ}$ cut, indicating that the degree of cutting affects biomechanical variables. Interestingly, the same results have also been found in men [41]. The question remains therefore if the increase is gender related.

Looking more in detail, all but two variables had fairly small differences in hip, knee and ankle angles, ranging from $0^{\circ}$ to $6.5^{\circ}$. Peak hip flexion (women, smaller) and peak knee valgus (women, greater) were found to have a relatively large gender difference of $10.9^{\circ}$ and $10.05^{\circ}$, respectively, in only two studies $[16,26]$. It is interesting to 
Table 5 Electromyographic activity

\begin{tabular}{|c|c|c|c|c|c|c|}
\hline Dependent variable & Task & $\begin{array}{l}\text { Mean } \pm \text { SD }(n) \\
\text { Women }\end{array}$ & $\begin{array}{l}\text { Mean } \pm \text { SD }(n) \\
\text { Men }\end{array}$ & $\begin{array}{l}\text { Gender } \\
\text { difference }\end{array}$ & $P$ value & $\begin{array}{l}\text { Effect size } \\
(95 \% \mathrm{CI})\end{array}$ \\
\hline \multicolumn{7}{|l|}{ Mean EMG (\% MVIC) } \\
\hline $\begin{array}{l}\text { Vastus lateralis- } \\
\text { preparatory phase }^{\mathrm{a}}\end{array}$ & $\begin{array}{l}\text { Sidestep cutting } \\
\left(60^{\circ}\right)\end{array}$ & $186.14 \pm 102.75$ & $129.36 \pm 63.30$ & -56.78 & $0.001 *$ & $-0.67(-1.29$ to -0.02$)$ \\
\hline $\begin{array}{l}\text { Rectus femoris- } \\
\text { preparatory phase }^{a}\end{array}$ & $\begin{array}{l}\text { Sidestep cutting } \\
\left(60^{\circ}\right)\end{array}$ & $80.25 \pm 38.46$ & $80.19 \pm 47.84$ & -0.06 & N/A & $0.00(-0.62$ to 0.62$)$ \\
\hline $\begin{array}{l}\text { Medial hamstrings- } \\
\text { preparatory phase }^{\mathrm{a}}\end{array}$ & $\begin{array}{l}\text { Sidestep cutting } \\
\left(60^{\circ}\right)\end{array}$ & $72.19 \pm 34.75$ & $77.46 \pm 57.63$ & 5.27 & N/A & $0.11(-0.51$ to 0.73$)$ \\
\hline $\begin{array}{l}\text { Lateral hamstrings-- } \\
\text { preparatory phase }^{\mathrm{a}}\end{array}$ & $\begin{array}{l}\text { Sidestep cutting } \\
\left(60^{\circ}\right)\end{array}$ & $172.29 \pm 64.43$ & $194.92 \pm 113.68$ & 22.63 & N/A & $0.24(-0.38$ to -0.86$)$ \\
\hline $\begin{array}{l}\text { Gluteus medius- } \\
\text { preparatory phase }^{\mathrm{a}}\end{array}$ & $\begin{array}{l}\text { Sidestep cutting } \\
\left(60^{\circ}\right)\end{array}$ & $78.53 \pm 45.42$ & $84.93 \pm 48.53$ & 6.4 & N/A & $0.14(-0.49$ to 0.75$)$ \\
\hline $\begin{array}{l}\text { Gluteus maximus- } \\
\text { preparatory phase }^{\mathrm{a}}\end{array}$ & $\begin{array}{l}\text { Sidestep cutting } \\
\left(60^{\circ}\right)\end{array}$ & $256.08 \pm 175.68$ & $301.35 \pm 264.17$ & 45.27 & N/A & $0.20(-0.42$ to 0.82$)$ \\
\hline \multicolumn{7}{|l|}{ Mean EMG (\% MVIC) } \\
\hline $\begin{array}{l}\text { Vastus lateralis_loading } \\
\text { phase }^{\mathrm{a}}\end{array}$ & $\begin{array}{l}\text { Sidestep cutting } \\
\left(60^{\circ}\right)\end{array}$ & $320.86 \pm 164.65$ & $188.85 \pm 61.60$ & -132.01 & $0.001 *$ & $-1.06^{\ddagger}(-1.70$ to -0.38$)$ \\
\hline $\begin{array}{l}\text { Rectus femoris-loading } \\
\text { phase }^{\mathrm{a}}\end{array}$ & $\begin{array}{l}\text { Sidestep cutting } \\
\left(60^{\circ}\right)\end{array}$ & $173.32 \pm 81.08$ & $136.73 \pm 63.46$ & -36.59 & N/A & $-0.50(-1.12$ to 0.14$)$ \\
\hline $\begin{array}{l}\text { Medial hamstrings- } \\
\text { loading phase }\end{array}$ & $\begin{array}{l}\text { Sidestep cutting } \\
\left(60^{\circ}\right)\end{array}$ & $130.22 \pm 92.68$ & $128.02 \pm 48.60$ & -2.2 & N/A & $-0.03(-0.65$ to 0.59$)$ \\
\hline $\begin{array}{l}\text { Lateral hamstrings- } \\
\text { loading phase } \mathrm{e}^{\mathrm{a}}\end{array}$ & $\begin{array}{l}\text { Sidestep cutting } \\
\quad\left(60^{\circ}\right)\end{array}$ & $210.57 \pm 85.13$ & $194.18 \pm 143.23$ & -16.39 & N/A & $-0.14(-0.76$ to 0.48$)$ \\
\hline $\begin{array}{l}\text { Gluteus medius-loading } \\
\text { phase }^{\mathrm{a}}\end{array}$ & $\begin{array}{l}\text { Sidestep cutting } \\
\quad\left(60^{\circ}\right)\end{array}$ & $173.30 \pm 80.62$ & $138.42 \pm 39.78$ & -34.88 & $0.013^{*}$ & -0.55 ( -1.17 to 0.09$)$ \\
\hline $\begin{array}{l}\text { Gluteus maximus-loading } \\
\text { phase }^{\mathrm{a}}\end{array}$ & $\begin{array}{l}\text { Sidestep cutting } \\
\quad\left(60^{\circ}\right)\end{array}$ & $194.73 \pm 110.52$ & $186.08 \pm 110.41$ & -8.65 & N/A & -0.08 ( -0.70 to 0.54$)$ \\
\hline \multicolumn{7}{|l|}{ STMF at IC (Hz) } \\
\hline Vastus lateralis ${ }^{\mathrm{b}}$ & $\begin{array}{l}\text { Unanticipated } \\
\text { cutting }\left(45^{\circ}\right)\end{array}$ & $79.00 \pm 19.97(15)$ & $99.34 \pm 21.08$ & 20.34 & $0.011^{*}$ & $0.99^{\dagger}(0.21$ to 1.71$)$ \\
\hline Vastus medialis ${ }^{\mathrm{b}}$ & $\begin{array}{l}\text { Unanticipated } \\
\text { cutting }\left(45^{\circ}\right)\end{array}$ & $88.83 \pm 21.38(15)$ & $112.78 \pm 25.97(15)$ & 23.95 & $0.010^{*}$ & $1.01^{\dagger}(0.22$ to 1.74$)$ \\
\hline Rectus femoris $^{\mathrm{b}}$ & $\begin{array}{l}\text { Unanticipated } \\
\text { cutting }\left(45^{\circ}\right)\end{array}$ & $85.04 \pm 29.02(15)$ & $80.58 \pm 28.85$ & -4.46 & 0.676 & -0.15 ( -0.87 to 0.57$)$ \\
\hline Biceps femoris ${ }^{\mathrm{b}}$ & $\begin{array}{l}\text { Unanticipated } \\
\text { cutting }\left(45^{\circ}\right)\end{array}$ & $61.75 \pm 24.14$ & $89.20 \pm 41.12(15)$ & 27.45 & $0.034 *$ & $0.81^{\dagger}(0.05$ to 1.54$)$ \\
\hline Semitendinosus $^{\mathrm{b}}$ & $\begin{array}{l}\text { Unanticipated } \\
\text { cutting }\left(45^{\circ}\right)\end{array}$ & $64.27 \pm 22.38$ & $72.46 \pm 43.14(15)$ & 8.19 & 0.519 & $0.24(-0.49$ to 0.95$)$ \\
\hline Lateral gastrocnemius ${ }^{\mathrm{b}}$ & $\begin{array}{l}\text { Unanticipated } \\
\text { cutting }\left(45^{\circ}\right)\end{array}$ & $92.51 \pm 53.32(15)$ & $100.41 \pm 45.12(15)$ & 7.9 & 0.665 & $0.16(-0.56$ to 0.87$)$ \\
\hline Medial gastrocnemius ${ }^{b}$ & $\begin{array}{l}\text { Unanticipated } \\
\text { cutting }\left(45^{\circ}\right)\end{array}$ & $85.75 \pm 32.04(15)$ & $86.82 \pm 45.05(15)$ & 1.07 & 0.777 & $0.03(-0.69$ to 0.74$)$ \\
\hline Tibialis anterior $^{\mathrm{b}}$ & $\begin{array}{l}\text { Unanticipated } \\
\text { cutting }\left(45^{\circ}\right)\end{array}$ & $112.49 \pm 30.25$ & $110.10 \pm 55.86(15)$ & -2.39 & 0.885 & $-0.05(-0.77$ to 0.66$)$ \\
\hline \multicolumn{7}{|l|}{ STMF integral prestance phase } \\
\hline Vastus lateralis $^{\mathrm{b}}$ & $\begin{array}{l}\text { Unanticipated } \\
\text { cutting }\left(45^{\circ}\right)\end{array}$ & $18.39 \pm 6.31(15)$ & $23.47 \pm 10.28(15)$ & 5.08 & 0.114 & $0.60(-0.15$ to 1.31$)$ \\
\hline Vastus medialis ${ }^{\mathrm{b}}$ & $\begin{array}{l}\text { Unanticipated } \\
\text { cutting }\left(45^{\circ}\right)\end{array}$ & $21.43 \pm 9.43(15)$ & $27.75 \pm 13.82(15)$ & 6.32 & 0.155 & $0.53(-0.21$ to 1.25$)$ \\
\hline Rectus femoris ${ }^{\mathrm{b}}$ & $\begin{array}{l}\text { Unanticipated } \\
\text { cutting }\left(45^{\circ}\right)\end{array}$ & $16.42 \pm 6.98$ & $17.49 \pm 14.19(15)$ & 1.07 & 0.796 & $0.10(-0.62$ to 0.81$)$ \\
\hline Biceps femoris ${ }^{b}$ & $\begin{array}{l}\text { Unanticipated } \\
\text { cutting }\left(45^{\circ}\right)\end{array}$ & $25.20 \pm 8.33(15)$ & $25.47 \pm 15.12(15)$ & 0.27 & 0.952 & $0.02(-0.69$ to 0.74$)$ \\
\hline
\end{tabular}


Table 5 continued

\begin{tabular}{|c|c|c|c|c|c|c|}
\hline Dependent variable & Task & $\begin{array}{l}\text { Mean } \pm \text { SD }(n) \\
\text { Women }\end{array}$ & $\begin{array}{l}\text { Mean } \pm \text { SD }(n) \\
\text { Men }\end{array}$ & $\begin{array}{l}\text { Gender } \\
\text { difference }\end{array}$ & $P$ value & $\begin{array}{l}\text { Effect size } \\
(95 \% \mathrm{CI})\end{array}$ \\
\hline Semitendinosus $^{\mathrm{b}}$ & Unanticipated cutting $\left(45^{\circ}\right)$ & $29.15 \pm 9.28(15)$ & $28.05 \pm 15.43(15)$ & -1.10 & 0.815 & $-0.09(-0.80$ to 0.63$)$ \\
\hline $\begin{array}{l}\text { Lateral } \\
\text { gastrocnemius }\end{array}$ & Unanticipated cutting $\left(45^{\circ}\right)$ & $24.87 \pm 17.12(15)$ & $27.13 \pm 16.47(15)$ & 2.26 & 0.716 & $0.13(-0.59$ to 0.85$)$ \\
\hline $\begin{array}{l}\text { Medial } \\
\text { gastrocnemius }\end{array}$ & Unanticipated cutting $\left(45^{\circ}\right)$ & $26.72 \pm 10.99(15)$ & $25.66 \pm 13.89(15)$ & -1.06 & 0.819 & $-0.08(-0.80$ to 0.63$)$ \\
\hline Tibialis anterior $^{\mathrm{b}}$ & Unanticipated cutting $\left(45^{\circ}\right)$ & $35.46 \pm 15.12(15)$ & $38.18 \pm 20.11$ & 2.72 & 0.679 & $0.15(-0.57$ to 0.87$)$ \\
\hline Vastus lateralis $^{\mathrm{b}}$ & Unanticipated cutting $\left(45^{\circ}\right)$ & $34.65 \pm 6.49(15)$ & $47.72 \pm 13.62(15)$ & 13.07 & $0.002 *$ & $1.23^{*}(0.42$ to 1.97$)$ \\
\hline Vastus medialis ${ }^{\mathrm{b}}$ & Unanticipated cutting $\left(45^{\circ}\right)$ & $38.55 \pm 12.60(15)$ & $54.00 \pm 14.78$ & 15.45 & $0.005^{*}$ & $1.13^{*}(0.33$ to 1.86$)$ \\
\hline Rectus femoris ${ }^{b}$ & Unanticipated cutting $\left(45^{\circ}\right)$ & $35.58 \pm 9.59(15)$ & $47.49 \pm 17.02(15)$ & 11.91 & $0.025^{*}$ & $0.86^{\star}(-0.09$ to 1.59$)$ \\
\hline Biceps femoris ${ }^{b}$ & Unanticipated cutting $\left(45^{\circ}\right)$ & $36.95 \pm 6.74(15)$ & $44.48 \pm 18.21$ & 7.53 & 0.144 & $0.55(-0.19$ to 1.26$)$ \\
\hline Semitendinosus ${ }^{\mathrm{b}}$ & Unanticipated cutting $\left(45^{\circ}\right)$ & $37.82 \pm 13.05(15)$ & $38.96 \pm 19.09(15)$ & 1.14 & 0.851 & $0.07(-0.65$ to 0.78$)$ \\
\hline $\begin{array}{l}\text { Lateral } \\
\text { gastrocnemius }\end{array}$ & Unanticipated cutting $\left(45^{\circ}\right)$ & $56.33 \pm 20.80(15)$ & $56.86 \pm 18.01(15)$ & 0.53 & 0.941 & $0.03(-0.69$ to 0.74$)$ \\
\hline $\begin{array}{l}\text { Medial } \\
\text { gastrocnemius }\end{array}$ & Unanticipated cutting $\left(45^{\circ}\right)$ & $56.65 \pm 14.62(15)$ & $59.19 \pm 21.41(15)$ & 2.54 & 0.708 & $0.14(-0.58$ to 0.85$)$ \\
\hline Tibialis anterior $^{\mathrm{b}}$ & Unanticipated cutting $\left(45^{\circ}\right)$ & $56.69 \pm 15.89(15)$ & $58.58 \pm 27.00(15)$ & 1.89 & 0.817 & $0.09(-0.63$ to 0.80$)$ \\
\hline \multicolumn{7}{|l|}{ Total intensity at IC } \\
\hline Vastus lateralis ${ }^{\mathrm{b}}$ & Unanticipated cutting $\left(45^{\circ}\right)$ & $0.20 \pm 0.11$ & $0.22 \pm 0.15(15)$ & 0.02 & 0.617 & $0.15(-0.57$ to 0.86$)$ \\
\hline Vastus medialis ${ }^{\mathrm{b}}$ & Unanticipated cutting $\left(45^{\circ}\right)$ & $0.23 \pm 0.11$ & $0.24 \pm 0.14$ & 0.01 & 0.819 & $0.08(-0.64$ to 0.79$)$ \\
\hline Rectus femoris ${ }^{\mathrm{b}}$ & Unanticipated cutting $\left(45^{\circ}\right)$ & $0.17 \pm 0.11(15)$ & $0.16 \pm 0.19(15)$ & -0.01 & 0.924 & $-0.06(-0.78$ to 0.65$)$ \\
\hline Biceps femoris ${ }^{\mathrm{b}}$ & Unanticipated cutting $\left(45^{\circ}\right)$ & $0.15 \pm 0.14$ & $(15)$ & 0.03 & 0.478 & $0.23(-0.49$ to 0.94$)$ \\
\hline Semitendinosus ${ }^{\mathrm{b}}$ & Unanticipated cutting $\left(45^{\circ}\right)$ & 012 & $0.15 \pm 0$ & 0.03 & 0.505 & $0.21(-0.52$ to 0.92$)$ \\
\hline $\begin{array}{l}\text { Lateral } \\
\text { gastrocnemius }\end{array}$ & Unanticipated cutting $\left(45^{\circ}\right)$ & $.08 \pm 0.06(15)$ & $0.14 \pm 0.13(15)$ & 0.06 & 0.099 & $0.59(-0.15$ to 1.31$)$ \\
\hline $\begin{array}{l}\text { Medial } \\
\text { gastrocnemius }\end{array}$ & Unanticipated cutting $\left(45^{\circ}\right)$ & $0.14 \pm 0.16(15)$ & $0.14 \pm 0.1$ & 0.00 & 0.954 & $0.00(-0.72$ to 0.72$)$ \\
\hline Tibialis anterior $^{\mathrm{b}}$ & Unanticipated cutting $\left(45^{\circ}\right)$ & $0.31 \pm 0.13(15)$ & $0.26 \pm 0.21$ & -0.05 & 0.479 & $-0.29(-1.00$ \\
\hline \multicolumn{7}{|c|}{ Timing of peak total intensity (\%) } \\
\hline Vastus lateralis ${ }^{\mathrm{b}}$ & Unanticipated cutting $\left(45^{\circ}\right)$ & $15.55 \pm 11.45(15)$ & $17.08 \pm 12.74(15)$ & 1.53 & 0.732 & $0.13(-0.59$ to 0.84$)$ \\
\hline Vastus medialis ${ }^{\mathrm{b}}$ & Unanticipated cutting $\left(45^{\circ}\right)$ & $16.53 \pm 12.16(15)$ & $15.75 \pm 12.12(15)$ & -0.78 & 0.862 & $-0.06(-0.78$ to 0.65$)$ \\
\hline Rectus femoris ${ }^{\mathrm{b}}$ & Unanticipated cutting $\left(45^{\circ}\right)$ & $18.52 \pm 9.33(15)$ & $17.08 \pm 10.83(15)$ & -1.44 & 0.700 & $-0.14(-0.86$ to 0.58$)$ \\
\hline Biceps femoris ${ }^{\mathrm{b}}$ & Unanticipated cutting $\left(45^{\circ}\right)$ & $-12.35 \pm 6.64(15)$ & $-7.12 \pm 5.54(15)$ & 5.23 & $0.026^{*}$ & $0.86^{*}(0.09$ to 1.58$)$ \\
\hline Semitendinosus ${ }^{\mathrm{b}}$ & Unanticipated cutting $\left(45^{\circ}\right)$ & $-15.15 \pm 6.23(15)$ & $-15.32 \pm 12.35(15)$ & -0.17 & 0.962 & $-0.02(-0.73$ to 0.70$)$ \\
\hline $\begin{array}{l}\text { Lateral } \\
\text { gastrocnemius }^{\mathrm{b}}\end{array}$ & Unanticipated cutting $\left(45^{\circ}\right)$ & $26.71 \pm 14.39(15)$ & $26.28 \pm 19.14(15)$ & -0.43 & 0.945 & $-0.03(-0.74$ to 0.69$)$ \\
\hline $\begin{array}{l}\text { Medial } \\
\text { gastrocnemius }\end{array}$ & Unanticipated cutting $\left(45^{\circ}\right)$ & $20.49 \pm 20.31(15)$ & $32.86 \pm 14.28(15)$ & 12.37 & 0.064 & $0.70(-0.05$ to 1.42$)$ \\
\hline Tibialis anterior $^{\mathrm{b}}$ & Unanticipated cutting $\left(45^{\circ}\right)$ & $20.33 \pm 16.66(15)$ & $1.61 \pm 14.27(15)$ & -18.72 & $0.003 *$ & $-1.21^{\ddagger}(-1.95$ to -0.4 \\
\hline
\end{tabular}

As a percentage of the cutting cycle in relation to IC, a negative percentage indicates that the peak TI occurred before IC, a positive percentage indicates a peak TI occurring post IC

$S D$ standard deviation, $S T M F$ short-time mean frequency

* Significant difference $(P<0.05)$

+ Large effect size (i.e. $\geq 0.80$ ); N/A, data not provided

* Large effect size (i.e. $\geq 0.80$ or $\geq-0.80$ )

${ }^{a}$ Hanson et al. [20]

b Beaulieu et al. [16]

note that the results of the knee valgus angle varied from study to study, conflicting results making it hard to substantiate evidence as often postulated. It can be argued whether the small gender differences in hip, knee and ankle angles are of any clinical relevance in relation to injury risk. For example, considering that knee joint mechanics 
are governed by a combination of underlying bony geometric, laxity and tissue factors (which themselves demonstrate a degree of sex dependence), it is questionable whether these $0^{\circ}$ to $6.5^{\circ}$ gender differences are truly representing increased injury risk in women, especially if one realizes the possible measurement error due to skin movement [42-44].

On kinetics, how could external peak valgus moments ranging from 0.006 to $0.63 \mathrm{Nm} / \mathrm{kg}$ [30-32] be the reason why women rupture their ACL more frequently than men, if at least $94-\mathrm{Nm}$ valgus load is needed to rupture an ACL [45]? The highest valgus torque possible for women based on these numbers is $47.94 \mathrm{Nm}(0.63 \mathrm{Nm} / \mathrm{kg} \times 76.1 \mathrm{~kg})$ [32], safely within the safe zone of $94 \mathrm{Nm}$. However these numbers are based on in vitro measurements, we do not know for sure how much load it takes to rupture the ACL in in vivo situations. Factors as e.g. notch width, ACL size, hormonal influences and the $3 \mathrm{D}$ force rates in relation to contact time need to be considered. Therefore, a combination of in vivo, in vitro and modeling techniques will lead to improved understanding of injury risk.

The SD of the hip adduction and knee valgus moments are quite large for both genders (Table 4), the variance in this case seems therefore not to be gender specific. More important is which strategy is used by an individual athlete to get the hip, knee and ankle joints in the right direction with respect to the GRF. The actual load at the knee is comprised of multiple factors, such as orientation of the leg and the GRF. Currently, it is not known whether there are optimal levels of variability and whether deviations from these optimal levels increase the risk of injury [46].

\section{Neuromuscular control}

There were only two studies of interest found on EMG patterns during running-and-cutting tasks $[16,20]$. The two studies provided EMG variables at seven different instances during the task. There was a significant gender difference in four out of the seven variables for the VL activity, with one time an $\mathrm{ES}<0.80$. Both men and women showed greater VL activity in two of the four variables. VM activity was significantly different between genders only two times, with women showing less VM activity in both variables (STMF at IC and STMF integral stance phase). Furthermore, RF activity was different only one time, with women showing less activity (STMF integral stance phase). Clearly, no statement can be made whether men or women show more pronounced quadriceps muscle activation during plant and cutting maneuvers. For the hamstrings, women showed less BF activity measured by STMF at IC and earlier BF activity measured by timing of peak total intensity (\%). The outcomes do not seem to be related to the type of study (Hanson vs. Beaulieu). Mean
EMG (\% MVIC) during the loading phase was found to be higher in women than in men [20]. Interestingly, EMG patterns of the quadriceps and hamstrings found in this review during plant and cut tasks are different than mostly found during purely 'sagittal directed' tasks like walking and vertical jumping in which quadriceps dominance and/ or less hamstring activity has been reported in women $[4$, 47]. Also, the EMG data in this review do not show the same results compared to a review by Hewett et al. [48]; they did report women to have lower gluteal activity and increased quadriceps activation. The tasks included in the current review are different than (some of) the tasks analyzed by Hewett et al. This may indicate that neuromuscular differences may depend on the tasks examined.

Potential reasons for inconclusive results

Based on this review, biomechanical and neuromuscular gender differences during plant and cutting maneuvers remains inconclusive. The outcomes of the studies reviewed varied, even though we selected a specific task to study. The studies did score fair to good on the methodological quality assessment, but there was methodological heterogeneity (Table 2) that might cause the lack of consensus across the studies. First, the sports level of the included subjects ranged from recreational level to NCAA Division I. Some studies included players at a variety of levels [16, 27, 31] or from a variety of sports [26, 27]. Considering the difference in epidemiology, gender differences should be analyzed in the same populations in which injuries occur.

In addition, different statistical methods were used and many studies had a low sample size (Table 2). Statistics and sample size have a great effect on results. The latter leads to low power and the risk of type II error. For example, if a difference of the means between two groups is considered clinically significant when it is greater than each group's standard deviation, at least 16 subjects are needed in each group to have $80 \%$ statistical power. Only the study by Hanson et al. had more than 16 subjects included in each group [20].

Different motion analysis systems were used to collect data, and tasks were performed differently in the included studies that could explain variety in outcome.

\section{Considerations and future research}

This review study gives an objective overview of current research available on neuromuscular and biomechanical gender difference during plant and cutting maneuvers. The question raises whether ACL injuries during plant and cutting maneuvers are gender related. Differences were found, but what do these small differences mean from a 
clinical point of view? Do they truly represent increased injury risk in women? Does it mean women have to move like men in order to reduce injury risk? The continued isolated focus on gender has recently been questioned [49]. In the 2008 report of Research Retreat on ACL injuries, it was stated that "it is time to move beyond the purely descriptive sex comparison studies that continue to dominate the literature and more critically examine the underlying causes for these differences and whether they truly reflect an increased injury risk for the physically active female". Even though those descriptive studies provide us with valuable information, cause-and-effect relationships are still not fully understood [9] and inferring injury risk from such assessments is questionable. The examination of biomechanical and neuromuscular contributions to injury risk should not be isolated and should extend beyond an isolated gender focus. We need to realize that men still have the largest number of ACL injuries [50]. Each gender may have their own risk factors [51]. Adding computer modeling and in vitro measurements will complement biomechanical studies and simulating what occurs during the injury event gives important information to identify high-risk athletes [49]. Once this is better understood, more specific and individualized prevention programs can be developed. Frontal plane valgus collapse as well as sagittal and transverse plane biomechanical and neuromuscular factors contribute to ACL injury [10, 52]. Recently, the LESS score was introduced to quantify multiplanar landing mechanisms that could aid in our understanding of specific athletes at high risk [53].

\section{Conclusion}

This review found that biomechanical and neuromuscular gender differences during cut and plant tasks show mainly small differences of which the clinical relevance can be questioned. However, it should be noted that the ES was inconsistent. This may indicate that future studies with higher statistical power may change the conclusions as drawn from the current review. It is therefore advised that research moves beyond the isolated gender comparison and that larger sample sizes will be included. Our results cannot be extrapolated to other type of tasks. This review adds to the literature as to how to improve on designing experiments to draw valid conclusions, in order to direct future ACL injury prevention programs.

Acknowledgments We thank Anita A. H. Verhoeven, MD, PhD, from the University Medical Center Groningen, the Netherlands, for her assistance in performing a thorough literature search strategy. We also thank Stephen Lyman, PhD from the Hospital for Special Surgery in New York and Cees P. van der Schans, PhD, PT from the Hanze University Groningen, Center for Research and Development in Health Care and Nursing for their assistance in data analysis. No financial support was received for this study, and no potential conflict of interest applies to this study.

Open Access This article is distributed under the terms of the Creative Commons Attribution Noncommercial License which permits any noncommercial use, distribution, and reproduction in any medium, provided the original author(s) and source are credited.

\section{References}

1. Arendt E, Dick R (1995) Knee injury patterns among men and women in collegiate basketball and soccer. NCAA data and review of literature. Am J Sports Med 23:694-701

2. Griffin LY, Agel J, Albohm MJ, Arendt EA, Dick RW, Garrett WE, Garrick JG, Hewett TE, Huston L, Ireland ML, Johnson RJ, Kibler WB, Lephart S, Lewis JL, Lindenfeld TN, Mandelbaum BR, Marchak P, Teitz CC, Wojtys EM (2000) Noncontact anterior cruciate ligament injuries: risk factors and prevention strategies. J Am Acad Orthop Surg 8:141-150

3. Gwinn DE, Wilckens JH, McDevitt ER, Ross G, Kao TC (2000) The relative incidence of anterior cruciate ligament injury in men and women at the United States Naval Academy. Am J Sports Med 28:98-102

4. Hewett TE, Myer GD, Ford KR (2006) Anterior cruciate ligament injuries in female athletes: Part 1, mechanisms and risk factors. Am J Sports Med 34:299-311

5. Oiestad BE, Engebretsen L, Storheim K, Risberg MA (2009) Knee osteoarthritis after anterior cruciate ligament injury: a systematic review. Am J Sports Med 37:1434-1443

6. Agel J, Arendt EA, Bershadsky B (2005) Anterior cruciate ligament injury in national collegiate athletic association basketball and soccer: a 13-year review. Am J Sports Med 33:524530

7. Griffin LY, Albohm MJ, Arendt EA, Bahr R, Beynnon BD, Demaio M, Dick RW, Engebretsen L, Garrett WE Jr, Hannafin JA, Hewett TE, Huston LJ, Ireland ML, Johnson RJ, Lephart S, Mandelbaum BR, Mann BJ, Marks PH, Marshall SW, Myklebust G, Noyes FR, Powers C, Shields C Jr, Shultz SJ, Silvers H, Slauterbeck J, Taylor DC, Teitz CC, Wojtys EM, Yu B (2006) Understanding and preventing noncontact anterior cruciate ligament injuries: a review of the Hunt Valley II meeting, January 2005. Am J Sports Med 34:1512-1532

8. Hewett TE, Myer GD, Ford KR, Heidt RS Jr, Colosimo AJ, McLean SG, van den Bogert AJ, Paterno MV, Succop P (2005) Biomechanical measures of neuromuscular control and valgus loading of the knee predict anterior cruciate ligament injury risk in female athletes: a prospective study. Am J Sports Med 33:492-501

9. Yu B, Garrett WE (2007) Mechanisms of non-contact ACL injuries. Br J Sports Med 41 Suppl 1:47-51

10. Alentorn-Geli E, Myer GD, Silvers HJ, Samitier G, Romero D, Lazaro-Haro C, Cugat R (2009) Prevention of non-contact anterior cruciate ligament injuries in soccer players. Part 1: mechanisms of injury and underlying risk factors. Knee Surg Sports Traumatol Arthrosc 17:705-729

11. Boden B, Dean G, Feagin JJ, Garrett WJ (2000) Mechanisms of anterior cruciate ligament injury. Orthopedics 23:573-578

12. Fauno P, Wulff Jakobsen B (2006) Mechanism of anterior cruciate ligament injuries in soccer. Int J Sports Med 27:75-79

13. Feagin J, Lambert K (1985) Mechanism of injury and pathology of anterior cruciate ligament injuries. Orthop Clin North Am $16: 41-45$ 
14. Olsen OE, Myklebust G, Engebretsen L, Bahr R (2004) Injury mechanisms for anterior cruciate ligament injuries in team handball: a systematic video analysis. Am J Sports Med 32:1002-1012

15. Cohen J (1988) Statistical power analysis for the behavioral sciences. Lawrence Erlbaum Associates, Hillsdale, NJ

16. Beaulieu M, Lamontagne M, Xu L (2008) Gender differences in time-frequency EMG analysis of unanticipated cutting maneuvers. Med Sci Sports Exerc 40:1795-1804

17. Bencke J, Naesborg H, Simonsen EB, Klausen K (2000) Motor pattern of the knee joint muscles during side-step cutting in European team handball. Influence on muscular co-ordination after an intervention study. Scand J Med Sci Sports 10:68-77

18. Colby S, Francisco A, Yu B, Kirkendall D, Finch M, Garrett W Jr (2000) Electromyographic and kinematic analysis of cutting maneuvers. Implications for anterior cruciate ligament injury. Am J Sports Med 28:234-240

19. Ford KR, Myer GD, Toms HE, Hewett TE (2005) Gender differences in the kinematics of unanticipated cutting in young athletes. Med Sci Sports Exerc 37:124-129

20. Hanson A, Padua D, Troy Blackburn J, Prentice W, Hirth C (2008) Muscle activation during side-step cutting maneuvers in male and female soccer athletes. J Athl Train 43:133-143

21. James CR, Sizer PS, Starch DW, Lockhart TE, Slauterbeck J (2004) Gender differences among sagittal plane knee kinematic and ground reaction force characteristics during a rapid sprint and cut maneuver. Res Q Exerc Sport 75:31-38

22. Landry SC, McKean KA, Hubley-Kozey CL, Stanish WD, Deluzio KJ (2007) Neuromuscular and lower limb biomechanical differences exist between male and female elite adolescent soccer players during an unanticipated run and crosscut maneuver. Am J Sports Med 35:1901-1911

23. Landry SC, McKean KA, Hubley-Kozey CL, Stanish WD, Deluzio KJ (2007) Neuromuscular and lower limb biomechanical differences exist between male and female elite adolescent soccer players during an unanticipated side-cut maneuver. Am J Sports Med 35:1888-1900

24. Malinzak RA, Colby SM, Kirkendall DT, Yu B, Garrett WE (2001) A comparison of knee joint motion patterns between men and women in selected athletic tasks. Clin Biomech (Bristol, Avon) 16:438-445

25. McLean SG, Huang X, van den Bogert AJ (2005) Association between lower extremity posture at contact and peak knee valgus moment during sidestepping: implications for ACL injury. Clin Biom 20:863-870

26. McLean SG, Lipfert SW, van den Bogert AJ (2004) Effect of gender and defensive opponent on the biomechanics of sidestep cutting. Med Sci Sports Exerc 36:1008-1016

27. McLean SG, Neal RJ, Myers PT, Walters MR (1999) Knee joint kinematics during the sidestep cutting maneuver: potential for injury in women. Med Sci Sports Exerc 31:959-968

28. Pollard C, Heiderscheit B, van Emmerik R, Hamill J (2005) Gender differences in lower extremity coupling variability during an unanticipated cutting maneuver. J Appl Biomech 21:143-152

29. Pollard C, Sigward S, Powers C (2007) Gender differences in hip joint kinematics and kinetics during side-step cutting maneuver. Clin J Sport Med 17:38-42

30. Pollard CD, Davis IM, Hamill J (2004) Influence of gender on hip and knee mechanics during a randomly cued cutting maneuver. Clin Biomech (Bristol, Avon) 19:1022-1031

31. Sigward SM, Powers CM (2006) The influence of gender on knee kinematics, kinetics and muscle activation patterns during sidestep cutting. Clin Biom 21:41-48

32. McLean SG, Huang X, van den Bogert AJ (2005) Association between lower extremity posture at contact and peak knee valgus moment during sidestepping: implications for ACL injury. Clin Biomech (Bristol, Avon) 20:863-870

33. von Tscharner V, Goepfert B (2003) Gender dependent EMGs of runners resolved by time/frequency and principal pattern analysis. J Elect Kinesiol 13:253-272

34. Altman DG, Schulz KF, Moher D, Egger M, Davidoff F, Elbourne D, Gøtzsche PC, Lang T (2001) The revised CONSORT statement for reporting randomized trials: explanation and elaboration. Ann Intern Med 134:663-694

35. Maher CG, Sherrington C, Herbert RD, Moseley AM, Elkins M (2003) Reliability of the PEDro scale for rating quality of randomized controlled trials. Phys Ther 83:713-721

36. Whiting P, Rutjes AWS, Reitsma JB, Bossuyt PMM, Kleijnen J (2003) The development of QUADAS: a tool for the quality assessment of studies of diagnostic accuracy included in systematic reviews. BMC Med Res Methodol 3:25

37. Kristianslund E, Krosshaug T (2010) Sidestep cutting technique and valgus knee loading. Paper presented at the ESSKA, Oslo, Norway

38. Cerulli G, Benoit DL, Lamontagne M, Caraffa A, Liti A (2003) In vivo anterior cruciate ligament strain behaviour during a rapid deceleration movement: case report. Knee Surg Sports Traumatol Arthrosc 11:307-311

39. Fung DT, Hendrix RW, Koh JL, Zhang LQ (2007) ACL impingement prediction based on MRI scans of individual knees. Clin Orthop Relat Res 460:210-218

40. Imwalle L, Myer G, Ford K, Hewett T (2009) The relationship between hip and knee kinematics in female athletes during cutting maneuvers: a possible link to non-contact ACL injury and prevention. J Strength Cond Res 23:2223-2230

41. Besier TF, Lloyd DG, Cochrane JL, Ackland TR (2001) External loading of the knee joint during running and cutting maneuvers. Med Sci Sports Exerc 33:1168-1175

42. Benoit DL, Ramsey DK, Lamontagne M, Xu L, Wretenberg P, Renstrom P (2006) Effect of skin movement artifact on knee kinematics during gait and cutting motions measured in vivo. Gait Posture 24:152-164

43. Reinschmidt C, van Den Bogert AJ, Murphy N, Lundberg A, Nigg BM (1997) Tibiocalcaneal motion during running, measured with external and bone markers. Clin Biomech (Bristol, Avon) 12:8-16

44. Reinschmidt C, van den Bogert AJ, Nigg BM, Lundberg A, Murphy N (1997) Effect of skin movement on the analysis of skeletal knee joint motion during running. J Biomech 30:729-732

45. McLean SG, Su A, van den Bogert AJ (2003) Development and validation of a 3-D model to predict knee joint loading during dynamic movement. J Biomech Eng 125:864-874

46. Shultz SJ, Schmitz RJ, Nguyen A (2008) Research Retreat IV: ACL injuries-the gender bias, April 3-5, 2008, Greensboro, NC. J Athl Train 43:530-537

47. Hewett TE, Myer GD, Ford KR, Heidt RS Jr, Colosimo AJ, McLean SG, van den Bogert AJ, Paterno MV, Succop P (2005) Biomechanical measures of neuromuscular control and valgus loading of the knee predict anterior cruciate ligament injury risk in female athletes: a prospective study. Am J Sports Med 33:492-501

48. Hewett TE, Zazulak BT, Myer GD, Ford KR (2005) A review of electromyographic activation levels, timing differences, and increased anterior cruciate ligament injury incidence in female athletes. Br J Sports Med 39:347-350

49. McLean SG (2008) The ACL injury enigma: we can't prevent what we don't understand. J Athl Train 43:538-540

50. Renstrom P, Ljungqvist A, Arendt E, Beynnon B, Fukubayashi T, Garrett W, Georgoulis T, Hewett TE, Johnson R, Krosshaug T, Mandelbaum B, Micheli L, Myklebust G, Roos E, Roos H, Schamasch P, Shultz S, Werner S, Wojtys E, Engebretsen L 
(2008) Non-contact ACL injuries in female athletes: an International Olympic Committee current concepts statement. $\mathrm{Br} \mathrm{J}$ Sports Med 42:394-412

51. Alentorn-Geli E, Myer GD, Silvers HJ, Samitier G, Romero D, Lazaro-Haro C, Cugat R (2009) Prevention of non-contact anterior cruciate ligament injuries in soccer players. Part 2: a review of prevention programs aimed to modify risk factors and to reduce injury rates. Knee Surg Sports Traumatol Arthrosc 17:859-879
52. Quatman CE, Hewett TE (2009) The anterior cruciate ligament injury controversy: is "valgus collapse" a sex-specific mechanism? Br J Sports Med 43:328-335

53. Padua DA, Marshall SW, Boling MC, Thigpen CA, Garrett WE Jr, Beutler AI (2009) The Landing Error Scoring System (LESS) Is a valid and reliable clinical assessment tool of jump-landing biomechanics: The JUMP-ACL study. Am J Sports Med 37:1996-2002 